\title{
Retrograde cerebral perfusion with intermittent pressure augmentation provides adequate neuroprotection: Diffusion- and perfusion-weighted magnetic resonance imaging study in an experimental canine model
}

Mitsuhiro Kawata, MD, ${ }^{a}$ Masaki Sekino, PhD, ${ }^{\mathrm{b}}$ Shinichi Takamoto, MD, PhD, ${ }^{a}$ Shoogo Ueno, PhD, ${ }^{\mathrm{b}}$ Sachiko Yamaguchi, MS, ${ }^{b}$ Kazuo Kitahori, MD, PhD, ${ }^{a}$ Hiroyuki Tsukihara, MD, ${ }^{a}$ Yoshihiro Suematsu, MD, PhD, ${ }^{a}$ Minoru Ono, MD, PhD, ${ }^{a}$ Noboru Motomura, MD, PhD, ${ }^{a}$ Tetsuro Morota, MD, PhD, and Arata Murakami, MD, PhD ${ }^{a}$

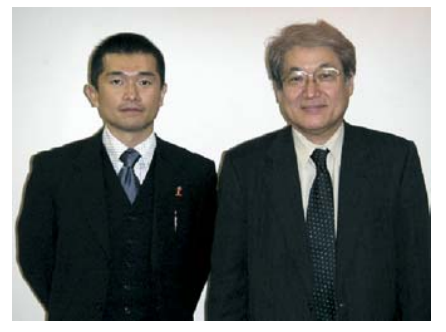

Drs Kawata and Takamoto (left to right)

From the Departments of Cardiothoracic Surgery $^{\mathrm{a}}$ and Biomedical Engineering, Bioimaging and Biomagnetics, ${ }^{\mathrm{b}}$ Graduate School of Medicine, University of Tokyo, Tokyo, Japan.

Received for publication Oct 24, 2005; revisions received March 6, 2006; accepted for publication March 28, 2006.

Address for reprints: Mitsuhiro Kawata, MD, Department of Cardiothoracic Surgery, Graduate School of Medicine, University of Tokyo, 7-3-1 Hongo, Bunkyo-ku, Tokyo 113-8655, Japan (E-mail: mkawataths@umin.ac.jp).

J Thorac Cardiovasc Surg 2006;132:933-40

$0022-5223 / \$ 32.00$

Copyright $(C) 2006$ by The American Association for Thoracic Surgery

doi:10.1016/j.jtcvs.2006.03.064
Objective: Diffusion- and perfusion-weighted magnetic resonance imaging can identify ischemic brain injury in the hyperacute stage. For neuroprotection during thoracic aortic surgery, we developed a novel retrograde cerebral perfusion with intermittent pressure augmentation. The purpose of this study was to assess the efficiency of this novel method for neuroprotection in real time by using diffusionand perfusion-weighted magnetic resonance imaging.

Methods: Sixteen beagle dogs were randomly divided into 4 groups: the antegrade selective cerebral perfusion group $(n=4$; antegrade selective cerebral perfusion at a flow rate of $\left.10 \mathrm{~mL} \cdot \mathrm{kg}^{-1} \cdot \min ^{-1}\right)$; the intermittent retrograde cerebral perfusion group $(n=4$; retrograde cerebral perfusion at a baseline pressure of $15 \mathrm{~mm} \mathrm{Hg}$ with intermittent pressure augmentation to $45 \mathrm{~mm} \mathrm{Hg}$ every 30 seconds); the conventional retrograde cerebral perfusion group $(n=4$; conventional retrograde cerebral perfusion at a fixed pressure of $25 \mathrm{~mm} \mathrm{Hg}$ ); and the circulatory arrest group $(\mathrm{n}=4$; only circulatory arrest). Diffusion- and perfusion-weighted magnetic resonance images were acquired during each session of cerebral perfusion. Regions of interest were defined, and the apparent diffusion coefficient and relative regional cerebral blood volume were calculated in these regions of interest. Finally, the brain was evaluated for its histopathologic damage score.

Results: The best apparent diffusion coefficient values were observed in the intermittent retrograde cerebral perfusion group in all the regions of interest, although the relative regional cerebral blood volume values were mostly lower than those in the antegrade selective cerebral perfusion group. The total Histopathologic Damage Score $(0$, normal; 32 , worst $)$ in the intermittent retrograde cerebral perfusion group $(8.0 \pm 0.6)$ was significantly lower than that in the conventional retrograde cerebral perfusion $(17.5 \pm 1.7 ; P<.01)$ and circulatory arrest $(25 \pm 1.0 ; P<0.01)$ groups and was equivalent to that in the antegrade selective cerebral perfusion group $(7.8 \pm$ $0.8 ; P=.9)$.

Conclusion: Intermittent retrograde cerebral perfusion provides adequate neuroprotection by allowing high apparent diffusion coefficient values to be maintained.

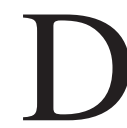
iffusion-weighted imaging (DWI) and perfusion-weighted imaging (PWI) are relatively new magnetic resonance imaging (MRI) techniques that allow identification of ischemic injury even in the hyperacute stage; that is, even before structural changes become evident on conventional brain MRI or computed tomography $(\mathrm{CT}){ }^{1-4}$ We developed a novel retrograde cerebral perfusion (RCP) method with intermittent pressure augmentation for cerebral protection during 


\section{Abbreviations and Acronyms \\ ADC = apparent diffusion coefficient \\ $\mathrm{ASCP}=$ antegrade selective cerebral perfusion \\ $\mathrm{CA}=$ circulatory arrest \\ $\mathrm{CPB}=$ cardiopulmonary bypass \\ DHCA = deep hypothermic circulatory arrest \\ DWI = diffusion-weighted magnetic resonance imaging \\ HDS = Histopathologic Damage Score \\ MRI = magnetic resonance imaging \\ PWI = perfusion-weighted magnetic resonance imaging \\ $\mathrm{RCP}=$ retrograde cerebral perfusion \\ $\mathrm{RCP}-\mathrm{C}=$ conventional retrograde cerebral perfusion \\ RCP-INT $=$ retrograde cerebral perfusion with intermittent pressure augmentation \\ $\mathrm{rrCBV}=$ relative regional cerebral blood volume}

thoracic aortic surgery. ${ }^{5}$ Intermittent augmentation of perfusion pressure effectively opens up cerebral vessels to allow adequate blood supply to the brain during hypothermia, thereby minimizing brain damage. ${ }^{5}$

The purpose of this study was to assess, by using diffusionweighted magnetic resonance imaging (DWI) and perfusionweighted magnetic resonance imaging (PWI), the extent of prevention of brain damage caused during deep hypothermic circulatory arrest (DHCA) by the use of RCP with intermittent pressure augmentation (RCP-INT) and to compare the neuroprotective efficiency of this method with that of antegrade selective cerebral perfusion (ASCP), conventional RCP (RCP-C), and circulatory arrest (CA) only (without cerebral perfusion). To the best of our knowledge, this is the first report of real-time assessment of the brain by DWI and PWI during aortic surgery.

\section{Materials and Methods}

Animal Care

This study was approved by the Animal Care and Use Committee of the University of Tokyo. All animals were acclimatized in the Section of Animal Research of the Center for Disease Biology and Integrative Medicine. All the animals received humane care in compliance with the Guide for the Care and Use of Laboratory Animals (Institute for Laboratory Animal Research, 1996).

\section{Experimental Groups}

Sixteen adult beagle dogs weighing 9 to $13 \mathrm{~kg}$ (mean, $10.6 \mathrm{~kg}$ ) were randomly divided into the following 4 groups: the ASCP group $\left(\mathrm{n}=4\right.$; ASCP at a flow rate of $10 \mathrm{~mL} \cdot \mathrm{kg}^{-1} \cdot \mathrm{min}^{-1}$ via an arterial cannula in the ascending aorta by clamping of the proximal ascending aorta, left subclavian artery, and descending aorta), the RCP-INT group $(n=4$; RCP via the maxillary veins of both sides at a baseline pressure of $15 \mathrm{~mm} \mathrm{Hg}$ with intermittent pressure augmentation to $45 \mathrm{~mm} \mathrm{Hg}$ every 30 seconds by clamping of the inferior vena cava), the RCP-C group ( $\mathrm{n}=4$; RCP-C via the maxillary veins of both sides at a fixed pressure of $25 \mathrm{~mm} \mathrm{Hg}$ by clamping of the inferior vena cava), or the CA group ( $n=4$; only CA without cerebral perfusion). The lower half of the body was not perfused in any of the groups. The CA group was examined both before surgery (body temperature $36^{\circ} \mathrm{C}$ ) and during standard cardiopulmonary bypass $\left(\mathrm{CPB}\right.$; body temperature $\left.18^{\circ} \mathrm{C}\right)$, as a control.

\section{Animal Preparation}

Anesthesia was induced in all animals by injection of ketamine hydrochloride $(10 \mathrm{mg} / \mathrm{kg}$ intramuscularly) and was maintained by injections of sodium pentobarbital throughout the operation. Endotracheal intubation was performed, and respiratory support was initiated with a pressure-controlled ventilator with $100 \%$ oxygen. The femoral artery and external jugular vein were cannulated with 20-gauge catheters for blood sampling, and the arterial and central venous pressures were monitored continuously. In the RCP-INT and RCP-C groups, two 16-gauge cannulas were inserted into the maxillary veins of both sides for RCP. The perfusion pressure used for the RCP was monitored in the distal maxillary veins, distal to the perfusion points. Blood samples were collected for arterial blood gas analysis, and measurements were performed of the base excess, serum electrolytes, hemoglobin, and oxygen saturation, with correction for the body temperature. The core temperature was monitored by using probes in the esophagus and rectum.

\section{Experimental Protocol}

Before commencement of the surgical preparation, the dogs in the CA group $(n=4)$ were placed in the supine position in a specially designed cradle and transferred into the magnetic resonance imaging (MRI) equipment. Preoperative DWI and PWI were conducted by using the methods described below under a body temperature condition of $36^{\circ} \mathrm{C}$.

At operation, a median sternotomy was performed; after systemic heparinization (300 IU/kg), a 10F arterial cannula (Medtronic Inc, Minneapolis, Minn) was inserted into the ascending aorta, and a 36F single venous cannula (Terumo Co, Ltd, Tokyo, Japan) was inserted into the right atrium. Extracorporeal circulation was accomplished by using a membrane oxygenator (Capiox RX-Baby RX; Terumo) and extracorporeal pump (TOW NOK heart-lung system, Compo III; Tonokura Ika Kogyo Co, Ltd, Tokyo, Japan) containing a special long circuit primed with $200 \mathrm{~mL}$ of homogeneous blood, $400 \mathrm{~mL}$ of a hemodilute solution of Ringer lactate solution, $50 \mathrm{~mL}$ of $20 \%$ human albumin, $20 \mathrm{~mL}$ of sodium bicarbonate, $100 \mathrm{~mL}$ of mannitol, and $5000 \mathrm{IU}$ of heparin. CPB was established at a flow rate of $100 \mathrm{~mL} \cdot \mathrm{kg}^{-1} \cdot \mathrm{min}^{-1}$, with the flow adjusted to maintain a mixed venous oxygen saturation of approximately $75 \%$. A 14-gauge catheter was inserted into the left ventricle via the apex to permit decompression of the left ventricle during the CPB. The animals were then cooled to $18^{\circ} \mathrm{C}$ by using a heat exchanger. The $\mathrm{pH}$ was maintained at 7.40 by using the $\mathrm{pH}$-stat principle, and the arterial $\mathrm{PaCO}_{2}$ was maintained at 35 to 40 $\mathrm{mm} \mathrm{Hg}$, corrected for body temperature. Cardiac arrest was induced with a cold cardioplegic solution after crossclamping of the ascending aorta. Then, the dogs in the CA group $(n=4)$ were transferred again into the MRI equipment. DWI and PWI were conducted under the standard $\mathrm{CPB}$ condition $\left(18^{\circ} \mathrm{C}\right)$. Then, all the animals were maintained in a state of DHCA for 120 minutes. 


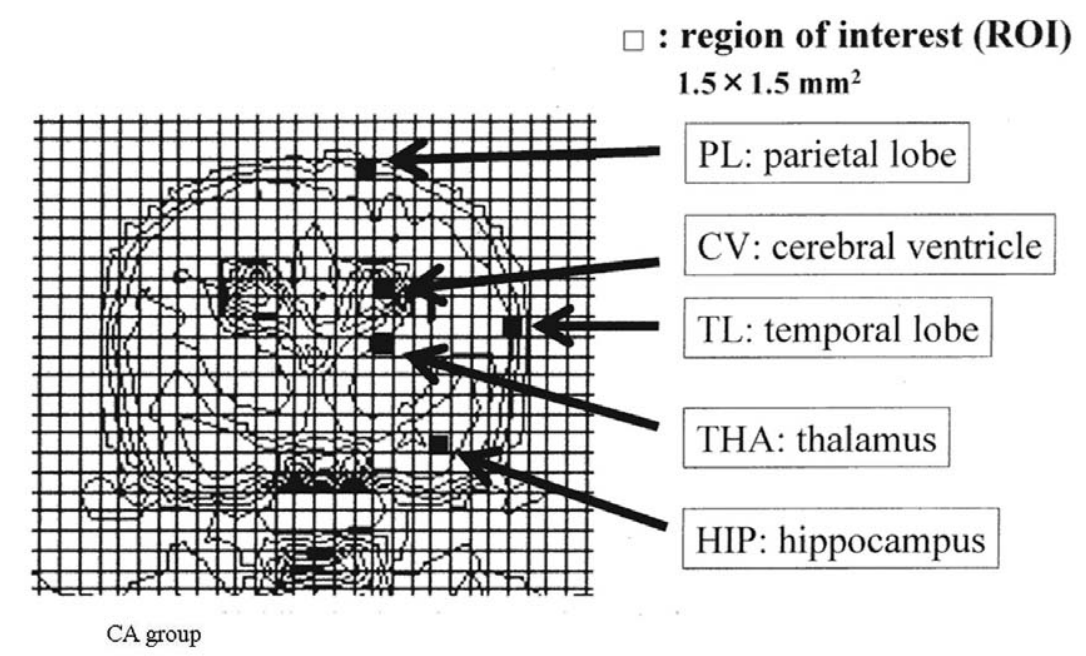

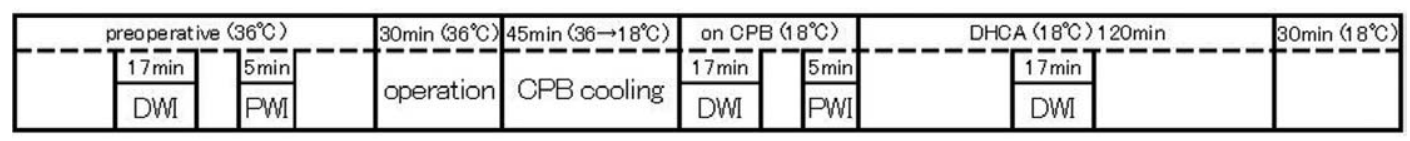

ASCP, RCP-INT, RCP-C group

\begin{tabular}{|c|c|c|c|c|c|}
\hline \multirow{3}{*}{ greoperative $\left(36^{\circ} \mathrm{C}\right.$} & $30 \min \left(36^{\circ} \mathrm{C}\right)$ & $45 \mathrm{~min}\left(36 \rightarrow 18^{\circ} \mathrm{C}\right)$ & \multicolumn{3}{|c|}{ Cerebral perfusion during DHCA $\left(18^{\circ} \mathrm{C}\right) 120 \mathrm{~min}\left(30 \mathrm{~min}\left(18^{\circ} \mathrm{C}\right)\right.$} \\
\hline & & & $17 \mathrm{~min}$ & $5 \mathrm{~min}$ & \\
\hline & Dotation & 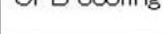 & DWI & PWI & \\
\hline
\end{tabular}

Figure 1. Region of interest (ROI). : $1.5 \times 1.5 \mathrm{~mm}^{2}$. PL, Parietal lobe; $C V$, cerebral ventricle; $T L$, temporal lobe; $T H A$, thalamus; HIP, hippocampus; CA, circulatory arrest; DWI, diffusion-weighted magnetic resonance imaging; $A S C P$, antegrade selective cerebral perfusion; $R C P$-INT, retrograde cerebral perfusion with intermittent pressure augmentation; $R C P-C$, conventional retrograde cerebral perfusion; $C P B$, cardiopulmonary bypass; $P W I$, perfusion-weighted magnetic resonance imaging. The time points of the experimental procedure in each group are shown.

During the DHCA, brain protection procedures were performed in each group according to the designated method, as described previously. In the RCP-INT and RCP-C groups, the arterial cannula in the ascending aorta was opened to maintain the common carotid arterial pressure equal to the atmospheric pressure. After the surgical preparation, the dogs were transferred into the MRI equipment.

\section{DWI and PWI}

Imaging was conducted in 4.7-T, 33-cm-bore MRI equipment (UNITY INOVA imaging spectrometer; Varian Associates Inc, Palo Alto, Calif) equipped with gradient coils consisting of specially designed 16-cm-diameter birdcage radiofrequency coil (transmit/receive). The position of the slices was determined from a sagittal brain image, and coronal imaging was chosen to include the hippocampus, thalamus, temporal lobe, parietal lobe, and cerebral ventricle, which were set as the regions of interest (Figure 1).

At first, DWI was obtained with a spin-echo pulse sequence (repetition time, 1000 milliseconds; echo time, 45 milliseconds) under the following imaging conditions: diffusion gradient $b$ values of 0 and $1000 \mathrm{~s} / \mathrm{mm}^{2}$ in the direction perpendicular to the sections, 10-mm-thick sections, $150-\mathrm{mm}$ field of view, and $128 \times$ 128 matrix. The acquisition time for the DWI was 17 minutes. $^{6}$ The DWI sequence generated an apparent diffusion coefficient (ADC) map for the section. The calculations were conducted by using Mathematica software, version 5.0 (Wolfram Research, Inc, Champaign, Ill). The dogs in the CA group $(n=4)$ were examined during $\mathrm{CPB}\left(18^{\circ} \mathrm{C}\right)$ and during $\mathrm{CA}\left(18^{\circ} \mathrm{C}\right)$.

Next, PWI was acquired by using the dynamic first pass of a $0.2 \mathrm{mmol} / \mathrm{kg}$ bolus of gadolinium-based contrast material (Gddiethylenetriaminepentaacetic acid [DTPA], Magnevist; Shering AG, Berlin, Germany) for the selected sections measured sequentially 300 times (acquisition time, 0.96 seconds for each measurement; repetition time, 15 milliseconds; echo time, 10 milliseconds). The bolus of contrast material was injected into the external jugular vein via the indwelling catheter (preoperative condition) or into the perfusion line of the CPB (operative condition), starting 1.5 minutes after the initiation of the sequence, followed by flushing with 
$10 \mathrm{~mL}$ of saline. The PWI data were obtained under the following conditions: 2-mm-thick sections, $150-\mathrm{mm}$ field of view, and $128 \times$ 64 matrix. Curve fitting was performed by using the MarquardtLevenberg algorithm. ${ }^{7}$ The area under the curve was calculated as a measure of the relative regional cerebral blood volume (rrCBV). ${ }^{8}$ The PWI sequence generated an rCBV map for the sections, and calculations were conducted by using Mathematica software, version 5.0. The dogs in the CA group ( $\mathrm{n}=4$ ) were examined during the $\mathrm{CPB}$ condition $\left(18^{\circ} \mathrm{C}\right)$; however, obviously, PWI was not performed during the CA. Sequential injections of large doses of Gd-DTPA contrast $(0.2 \mathrm{mmol} / \mathrm{kg})$ in the same animal in the CA group may affect the subsequent PWI. Therefore, the rrCBV value, the area under the curve, was calculated after adjustment for the effect of the accumulated Gd-DTPA contrast. Figure 1 clarifies the time points of the experimental procedure and the time points at which the DWI and PWI were performed in each group.

\section{Histopathologic Examination}

After the MRI data were obtained over almost 150 minutes after the induction of DHCA, the experiment was terminated for histopathologic studies. The brains were quickly harvested under deep hypothermia and adequate anesthesia and were fixed in $7 \%$ buffered formaldehyde solution. Five-millimeter-thick coronal sections through the entire brain were examined for gross lesions, and the sections through the following regions were especially closely examined: hippocampus, thalamus, temporal lobe, and parietal lobe. These sections were embedded in paraffin, cut to a thickness of $10 \mu \mathrm{m}$, stained with hematoxylin-eosin, and examined under a light microscope by a pathologist who was unaware of the experimental grouping. During this early period after the potential onset of hypoxic ischemic injury, the minimum criteria for the diagnosis of ischemic neuronal damage included mild cytoplasmic eosinophilia, shrunken neurons with scalloping of the margins, and nuclear changes consisting of coarsening of the nuclear chromatin or pyknosis. ${ }^{9-12}$ The modified Histopathologic Damage Score (HDS $)^{13}$ was calculated to determine the severity of the histopathologic damage. The scoring was defined as follows: no damaged neurons (0), minimal (2), mild (4), moderate (6), and severe (8).

\section{Statistical Analysis}

All data are presented as mean \pm SEM. Data were assessed by 1-way analysis of variance for comparisons among groups, followed by post hoc Dunnett tests. The Spearman rank order correlation coefficient was calculated to determine the correlations between the ADC values and the HDS. Differences between groups were considered statistically significant when the $P$ value was less than .05 . All statistics were computed by using the JMP analysis program, version 5.1 (SAS Institute Inc, Cary, $\mathrm{NC})$.

\section{Results}

\section{Diffusion-weighted MRI}

In the CA group, the ADC values in the hippocampus, thalamus, temporal lobe, and parietal lobe were significantly lower under the hypothermic condition $\left(18^{\circ} \mathrm{C}\right)$ during $\mathrm{CPB}$ than in the preoperative state $\left(36^{\circ} \mathrm{C} ; P=.02, .008, .03\right.$, and .006 , respectively). The ADC values in the hippocampus and thal-

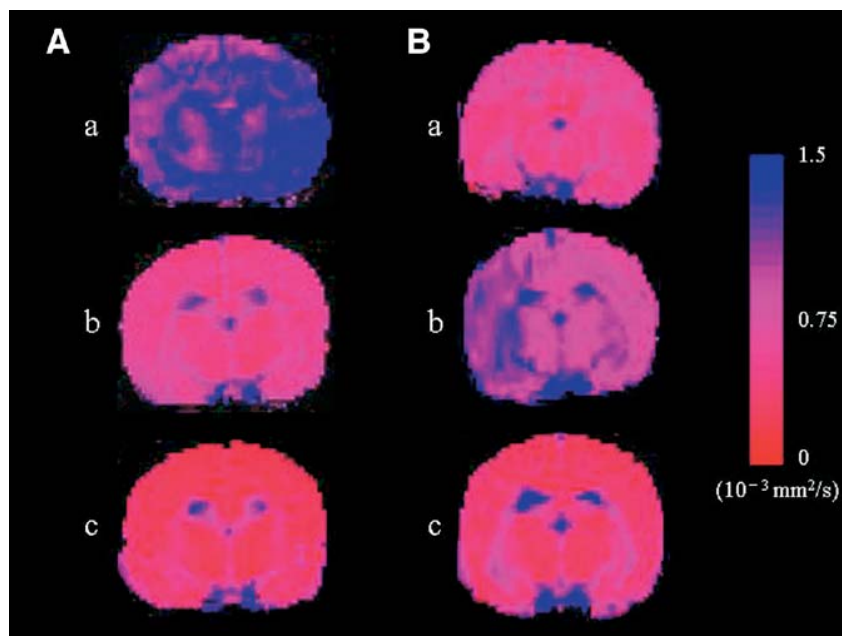

Figure 2. A, Apparent diffusion coefficient (ADC) map in the circulatory arrest group. a, Before surgery $\left(36^{\circ} \mathrm{C}\right)$; b, on cardiopulmonary bypass $\left(18^{\circ} \mathrm{C}\right)$; c, circulatory arrest $\left(18^{\circ} \mathrm{C}\right)$. B, ADC map for comparison among the different cerebral protection methods. a, Antegrade selective cerebral perfusion; $b$, retrograde cerebral perfusion with intermittent pressure augmentation; c, conventional retrograde cerebral perfusion.

amus were significantly lower during $\mathrm{CA}\left(18^{\circ} \mathrm{C}\right)$ than during $\mathrm{CPB}\left(18^{\circ} \mathrm{C} ; P=.018\right.$ and .038 , respectively). However, there were no significant differences in the cerebral ventricles between the 2 conditions (Figures 2, A, and 3,A). During DHCA, significantly higher ADC values were observed in the RCP-INT group than in the CA group in the hippocampus, thalamus, and temporal lobe $(P=.008, .003$, and .011 , respectively). In addition, the ADC values in the thalamus were also significantly higher in the ASCP group than in the CA group $(P=.013$; Figures $2, B$, and $3, B)$.

\section{Perfusion-weighted MRI}

Significantly higher rrCBV values in the temporal lobe were observed during hypothermia under $\mathrm{CPB}\left(18^{\circ} \mathrm{C}\right)$ than under the preoperative condition $\left(36^{\circ} \mathrm{C}\right)$ in the $\mathrm{CA}$ group $(P=.03$; Figures $4, A$, and 5, $A$ ). Conversely, the best $\operatorname{rrCBV}$ values in all the regions of interest during DHCA were observed in the ASCP group (Figures 4, B, and 5, B).

\section{Histopathologic Examination}

No macroscopic gross lesions were observed in any of the brain specimens. The total HDS did not differ significantly between the RCP-INT and ASCP groups (RCP-INT, $8 \pm$ 0.6; ASCP, $7.8 \pm 0.8 ; P=.99)$. However, the total HDS was significantly lower in the RCP-INT group than in the RCP-C and CA groups $(P<.001$ and $P<.001$, respectively). The regional HDS values for the 4 anatomic areas were also equivalent to the total HDS (Figure 6). 

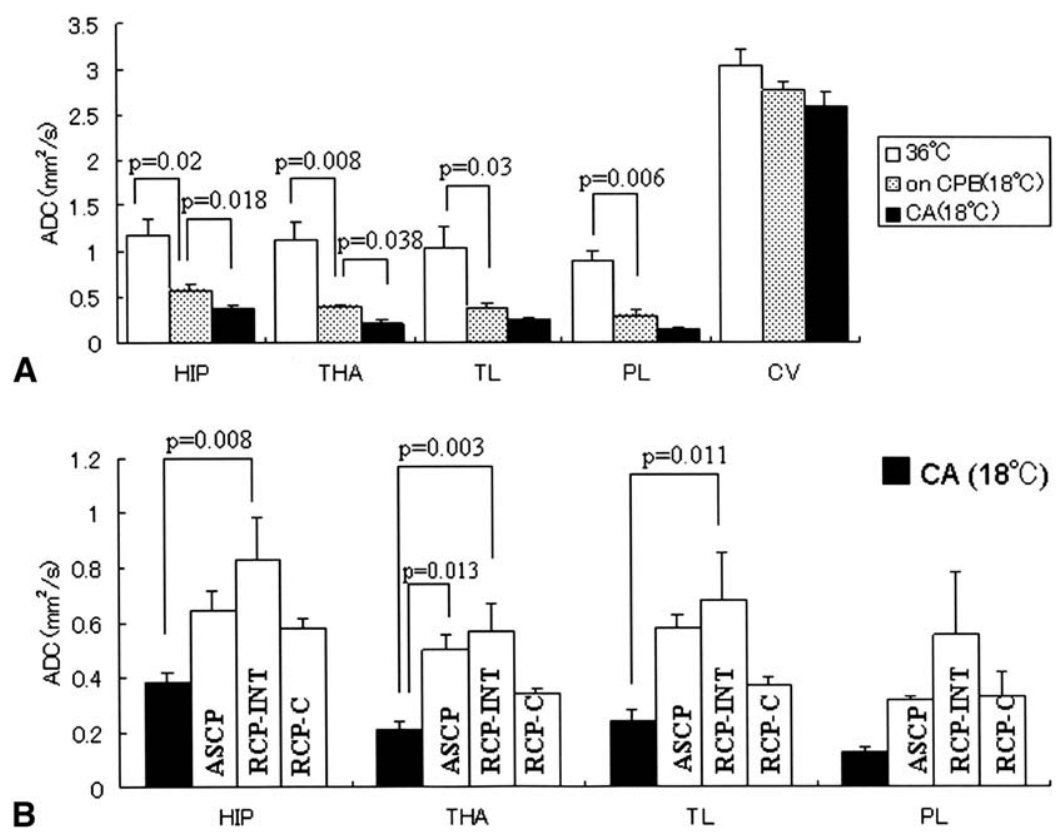

Figure 3. A, Apparent diffusion coefficient (ADC) values in the circulatory arrest (CA) group. HIP, Hippocampus; $T H A$, thalamus; $T L$, temporal lobe; $P L$, parietal lobe; $C V$, cerebral ventricle (cerebrospinal fluid); $C P B$, cardiopulmonary bypass. B, Comparison of the ADC values among the different cerebral protection methods. ASCP, Antegrade selective cerebral perfusion; $R C P-I N T$, retrograde cerebral perfusion with intermittent pressure augmentation; $\boldsymbol{R C P}-\boldsymbol{C}$, conventional retrograde cerebral perfusion.
Significant negative correlations between the ADC and the HDS were observed in regional brain areas (hippocampus, thalamus, and temporal lobe; Spearman correlation coefficient: hippocampus, $r=-0.7038, P=.0023$; thalamus, $r=-0.8198, P=.0001$; temporal lobe, $r=-0.8789$, $P<.001)$. A weak negative correlation was observed between the ADC and the HDS in the parietal lobe (Spear-

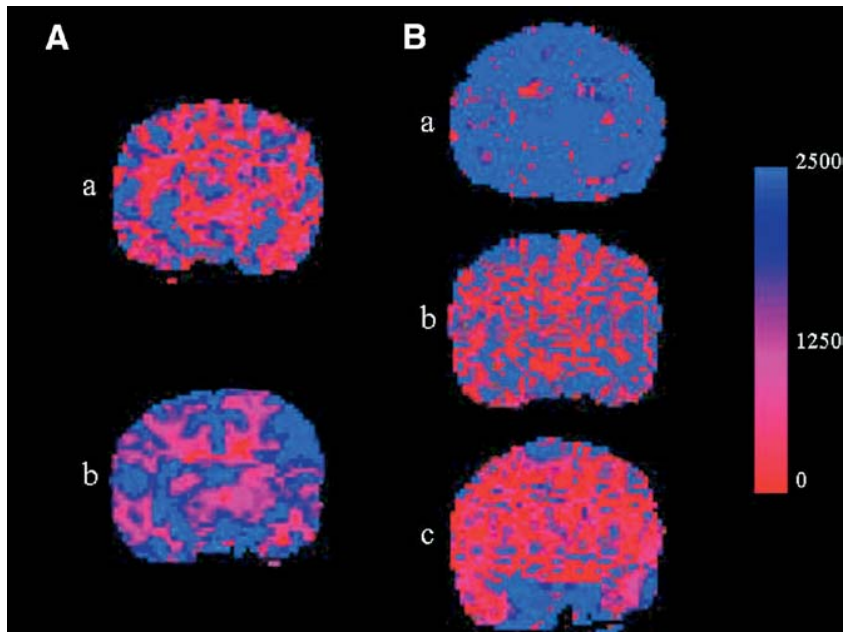

Figure 4. A, Relative regional cerebral blood volume (rrCBV) map in the circulatory arrest group. a, Before surgery $\left(36^{\circ} \mathrm{C}\right)$; $b$, on cardiopulmonary bypass $\left(18^{\circ} \mathrm{C}\right)$. $\mathrm{B}$, rrCBV map for comparison among the different cerebral protection methods. a, Antegrade selective cerebral perfusion; $b$, retrograde cerebral perfusion with intermittent pressure augmentation; c, conventional retrograde cerebral perfusion. man correlation coefficient: $r=-0.4352, P=.0920$ ). Examination of samples of hippocampus obtained from each group showed minimal evidence of cellular damage in the RCP-INT and ASCP groups (Figure 7), whereas moderate to severe ischemic neuronal change (shrunken neuron and nuclear pyknosis) was observed in the RCP-C and CA groups.

\section{Discussion}

DWI measures the restriction of water movements associated with failure of the cellular energy release mechanisms, membrane dysfunction, and cytotoxic edema and is sensitive to ischemia within minutes of its onset. ${ }^{6}$ Under the ischemic condition, the failure of adenosine triphosphatedependent ion pumps results in ischemic cellular depolarization and a water shift from the extracellular to the intracellular space. As a result of this decrease in extracellular water, diffusion is restricted in the extracellular space, thus resulting in signal decreases in the ADC, a quantitative measure of water diffusion. ${ }^{15}$ Lesions in the acute and hyperacute stages of infarction have low ADC values. ${ }^{8,14,15}$ PWI allows assessment of alterations of perfusion during acute ischemia. ${ }^{1}$ Details of the theory and methodology of the perfusion map calculations have been described in detail in previous articles. ${ }^{8,16}$ Recent studies have shown that in the acute phase of stroke, the area of decreased rrCBV is generally larger than the ischemic area, as seen in DWI., It is believed that measurement of the so-called perfusiondiffusion mismatch, defined as the difference between the large abnormalities in the rrCBV maps and the small abnormal areas on the ADC maps, is highly accurate for predicting enlarge- 


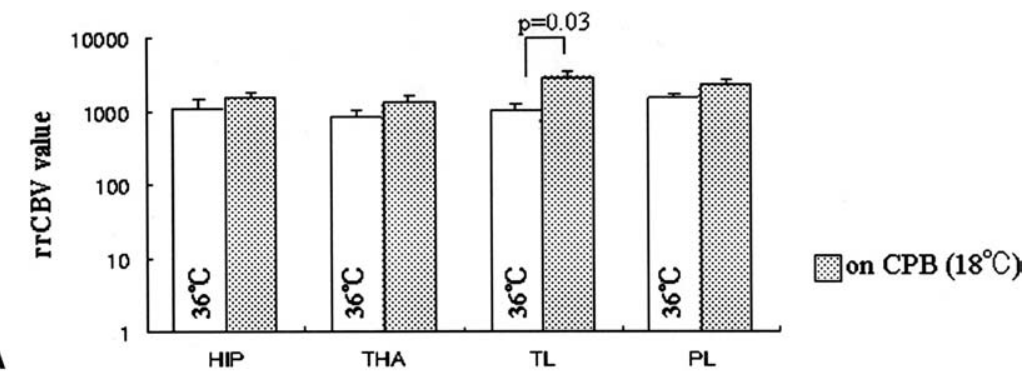

A
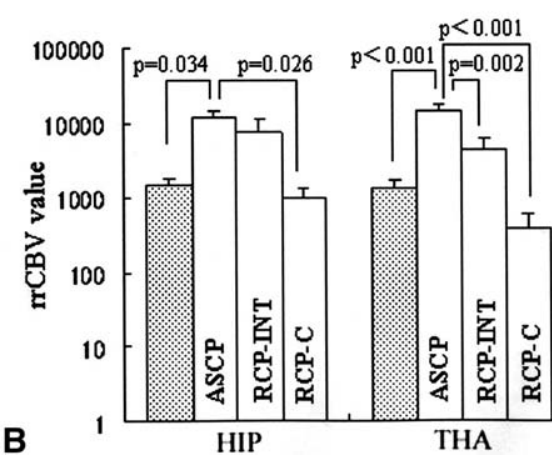

THA$$
\text { ( }
$$

$p<\frac{p<0.001}{p}<0.001 \frac{p}{p}<0.001$

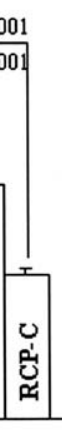

TL
Figure 5. A, Relative regional cerebral blood volume (rrCBV) values in the circulatory arrest group. HIP, Hippocampus; THA, thalamus; $T L$, temporal lobe; $P L$, parietal lobe; $C P B$, cardiopulmonary bypass. $B$, Comparison of the rrCBV values among the different cerebral protection methods. ASCP, Antegrade selective cerebral perfusion; $R C P$-INT, retrograde cerebral perfusion with intermittent pressure augmentation; $\boldsymbol{R C P}-\boldsymbol{C}$, conventional retrograde cerebral perfusion. ment of the lesion volume. ${ }^{8}$ The perfusion-diffusion mismatch area can be thought of as the ischemic penumbra, which is ischemic but still viable tissue surrounding the ischemic core.

Schaefer and colleagues ${ }^{1}$ reported that DWI was highly sensitive for the detection of acute ischemic stroke and that it was more sensitive than $\mathrm{rCBV}$. In this study, the rrCBV was lower at $36^{\circ} \mathrm{C}$ physiologic condition than at $18^{\circ} \mathrm{C}$ special condition, ASCP. This phenomenon was described by $\mathrm{Ye}$ and colleagues. ${ }^{10}$ The rrCBV during RCP-INT was also higher than that measured during preoperative at $36^{\circ} \mathrm{C}$. In addition, the $\operatorname{rrCBV}$ was lower during $\mathrm{CPB}$ under deep hypothermia than during ASCP and RCP-INT. We did not scientifically clarify the mechanism of this phenomenon. Further studies are needed. We did not think that rrCBV always correlated well with ADC.

Taking into consideration the histopathologic examination results, our data suggest that RCP-INT provides adequate neuroprotection during DHCA by allowing high ADC values to be maintained in the brain. Negative correlations between the ADC values and the HDS were observed in some regions of the brain. These results suggest that maintenance of high ADC values during DHCA may reflect decreased ischemic neuronal changes. We consider that the maintenance of the high ADC values in the RCP-INT group during DHCA may be related to the microcirculation. Friesenecker and colleagues ${ }^{17}$ concluded that the vasoconstrictive condition increases the arteriolar wall oxygen consumption and reduces the oxygen supply to the tissues. Shibata and associates ${ }^{18}$ reported that the vasodilated condition decreases the arteriolar wall oxygen consumption and increases the oxygen supply to the surrounding tissues. Therefore, we speculate that if adequate perfusion can be maintained via the veins, RCP can have beneficial effects on the brain tissue during DHCA, a vasoconstrictive condition.

However, we must also consider the effects of temperature on the ADC. Micromolecular diffusion is altered in

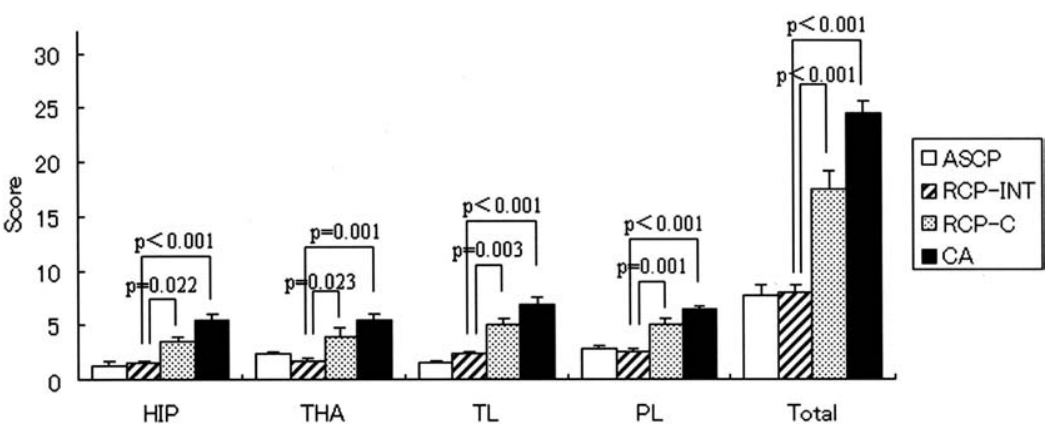

Figure 6. Histopathologic Damage Score. The score was defined as follows: no damaged neurons (0), minimal (2), mild (4), moderate (6), or severe (8). HIP, Hippocampus; $T H A$, thalamus; $T L$, temporal lobe; $P L$, parietal lobe; Total, total Histopathologic Damage Score; $A S C P$, antegrade selective cerebral perfusion; RCP-INT, retrograde cerebral perfusion with intermittent pressure augmentation; $\boldsymbol{R C P}-\boldsymbol{C}$, conventional retrograde cerebral perfusion; $C A$, circulatory arrest. The $P$ values shown present the results of the post hoc Dunnett test versus the result in the RCP-INT group. 

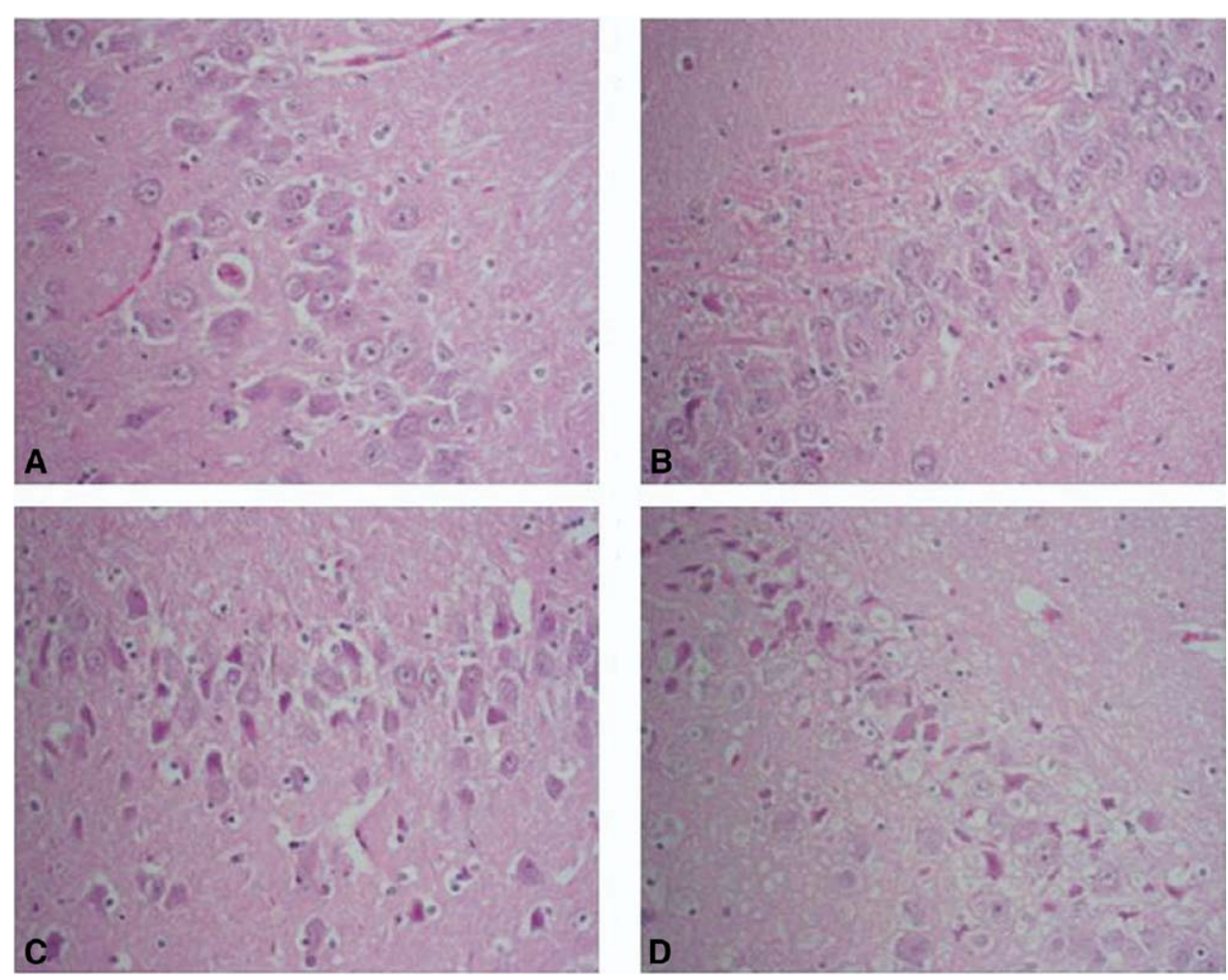

Figure 7. Sections of the hippocampus (stain, hematoxylineosin; original magnification, $200 \times$ ). A, Antegrade selective cerebral perfusion group. $B$, Retrograde cerebral perfusion with intermittent pressure augmentation group. C, Conventional retrograde cerebral perfusion group. D, Circulatory arrest group. Minimal evidence of cellular damage is seen in $A$ and $B$, whereas pyknotic nuclei, shrunken eosinophilic cytoplasm, and microvacuolization are seen in C and $\mathbf{D}$. association with temperature changes. Yenari and associates $^{19}$ reported that the $\mathrm{ADC}$ values varied directly and linearly with temperature. A $1^{\circ} \mathrm{C}$ change in the brain temperature corresponded to a $1.6 \%$ change in the ADC. It is considered that for the same body temperature $\left(18^{\circ} \mathrm{C}\right)$, an appropriate method for comparison of the efficiency of the various cerebral protection methods may be assessment of the ADC values on DWI.

Although our study groups were small, we conducted this experiment very carefully to prevent bias. Because the results of the study revealed significant differences in several characteristics among the groups, we decided that there may be no need to increase the size of the study samples. We support the $3 R$ s principle-replacement, reduction, and refinement - that has proven to be a common ground for research workers using animals.

We agree that the dog is not the best model for the study of RCP because dogs have multiple valves in the small internal jugular veins. However, an adult pig model is too large to be subjected to MRI in our high-magnetic-field (4.7-T), 33-cm-bore MRI equipment (UNITY INOVA) for animal experiments. Therefore, we decided to use an adult beagle dog model.

\section{Conclusion}

Our novel RCP method with intermittent pressure augmentation allowed maintenance of high ADC values in the brain and was therefore concluded as having the ability to provide adequate neuroprotection during DHCA. ${ }^{2,4}$

We thank Nobutaka Furuya, Takashi Kubota (laboratory assistant at the Department of Cardiothoracic Surgery), and Shigeo Saito (BioScience, Baxter Limited) for their technical assistance; Takuya Yamada, Wataru Yamanouchi, and Shogo Sanpei (Senko Medical Instrument Mfg Co, Ltd) for their medical engineering support; and Shigeki Aoki and Osamu Abe (Division of Radiology and Biomedical Engineering, Graduate School of Medicine, University of Tokyo) for their assistance in the image analysis.

\section{References}

1. Schaefer PW, Hunter GJ, He J, Hamberg LM, Sorensen AG, Schwamm LH, et al. Predicting cerebral ischemic infarct volume with diffusion and perfusion MR imaging. Am J Neuroradiol. 2002; 23:1785-94

2. Sorensen A, Buonanno F, Gonzales R, Schwamm L, Lev M, HuangHellinger F, et al. Hyperacute stroke: evaluation with combined multisection diffusion-weighted echo-planar MR imaging. Radiology. 1996;199:391-401.

3. Rordorf G, Koroshetz WJ, Copen WA, Cramer SC, Schaefer PW, Budzik RF, et al. Regional ischemia and ischemic injury in patients with acute middle cerebral artery stroke as defined by early diffusionweighted and perfusion-weighted MRI. Stroke. 1998;29:939-43.

4. Røhl L, Østergaard L, Simonsen CZ, Vestergaard PP, Andersen G, Sakoh M, et al. Viability thresholds of ischemic penumbra of hyperacute stroke defined by perfusion-weighted MRI and apparent diffusion coefficient. Stroke. 2001;32:1140-6.

5. Kitahori K, Takamoto S, Takayama H, Suematsu Y, Ono M, Motomura N, et al. A novel protocol of retrograde cerebral perfusion 
with intermittent pressure augmentation for brain protection. $J$ Thorac Cardiovasc Surg. 2005;130:363-70.

6. Arenillas JF, Rovira A, Molina C, Grive E, Montaner J, Alvarez-Sabin $\mathrm{J}$. Prediction of early neurological deterioration using diffusion- and perfusion-weighted imaging in hyperacute middle cerebral artery ischemic stroke. Stroke. 2002;33:2197-205.

7. Marquardt DW. An algorithm for least-squares estimations of nonlinear parameters. J Soc Indust Appl Math. 1963;11:431-41.

8. Kluytmans M, van Everdingen KJ, Kappelle LJ, Ramos LM, Viergever MA, van der Grond J. Prognostic value of perfusion- and diffusion-weighted MR imaging in first 3 days of stroke. Eur Radiol. 2000;10:1434-41.

9. Ye J, Yang L, Del Bigio MR, Filgueriras CL, Ede M, Summers R, et al. Neuronal damage after hypothermic circulatory arrest and retrograde cerebral perfusion in the pig. Ann Thorac Surg. 1996;61:1316-22.

10. Ye J, Ryner LN, Kozlowski P, Yang L, Del Bigio MR, Sun J, et al. Retrograde cerebral perfusion results in flow distribution abnormalities and neuronal damage. A magnetic resonance imaging and histopathological study in pigs. Circulation. 1998;98(suppl 2):II313-8.

11. Weller RO. General pathology of neurons: In: Symmers WSC, editor. Systemic pathology. Volume 4: Nervous system, muscle and eyes. 3rd ed. New York: Churchill Livingstone; 1990. p. 38-40.

12. Frosch MP, Anthony DC, Girolami UD. The central nervous system. In: Kumar V, Abbas AK, Fausto N, editors. Robins and Cotran pathologic basis of disease. 7th ed. Philadelphia: Elsevier Saunders; 2005. p. 1347-63.
13. Safer P, Xiao F, Radovsky A, Tanigawa K, Ebmeyer U, Bircher N, et al. Improved cerebral resuscitation from cardiac arrest in dogs with mild hypothermia plus blood flow promotion. Stroke. 1996; 27:105-13.

14. Warach S, Dashe JF, Edelman RR. Clinical outcome in ischemic stroke predicted by early diffusion-weighted and perfusion magnetic resonance imaging: a preliminary analysis. J Cereb Blood Flow Metab. 1996;16:53-9.

15. Ueda T, Yuh WTC, Maley JE, Quets JP, Hahn PY, Magnotta VA. Outcome of acute ischemic lesions evaluated by diffusion and perfusion MR imaging. Am J Neuroradiol. 1999;20:983-9.

16. Sorensen AG, Copen WA, Ostegaard L, Buonanno FS, Gonzalez RG, Rordorf G, et al. Hyperacute stroke: simultaneous measurement of relative cerebral blood volume, relative cerebral blood flow, and mean tissue transit time. Radiology. 1999;210:519-27.

17. Friesenecker B, Tsai AG, Dunser MW, Mayr AJ, Martini J, Knotzer H, et al. Oxygen distribution in microcirculation after arginine vasopressininduced arteriolar vasoconstriction. Am J Physiol Heart Circ Physiol. 2004;287:H1792-800.

18. Shibata M, Ichioka S, Kamiya A. Estimating oxygen consumption rates of arteriolar walls under physiological conditions in rat skeletal muscle. Am J Physiol Heart Circ Physiol. 2005;289:H295-300.

19. Yenari MA, Onley D, Hedehus M, deCrespigny A, Sun GH, Moseley $\mathrm{ME}$, et al. Diffusion- and perfusion-weighted magnetic resonance imaging of focal cerebral ischemia and cortical spreading depres sion under conditions of mild hypothermia. Brain Res. 2000;885: 208-19. 